\title{
The combination of arsenic, interferon-alpha, and zidovudine restores an "immunocompetent-like" micro-environment in patients with adult T-cell leukemia lymphoma
}

\author{
Ghada Kchour ${ }^{1}$, SA Rahim Rezaee ${ }^{2}$, Reza Farid ${ }^{3}$, Akram Ghantous ${ }^{4}$, Houshang Rafatpana ${ }^{3}$, Mahdi Tarhini ${ }^{5}$, \\ Mohamad-Mehdi Kooshyar ${ }^{6}$, Hiba El Hajj ${ }^{7}$, Fadwa Berry ${ }^{1}$, Roudaina Nasser ${ }^{7}$, Abbas Shirdel ${ }^{6}$, Zeina Dassouki ${ }^{7}$, \\ Mohamad Ezzedine ${ }^{1}$, Hossein Rahimi ${ }^{6}$, Ardeshir Ghavamzadeh ${ }^{8}$, Hugues de Thé ${ }^{9}$ Olivier Hermine ${ }^{10}$, \\ Mahmoud Mahmoudi ${ }^{3}$, Ali Bazarbachi ${ }^{{ }^{*}}$
}

From 16th International Conference on Human Retroviruses: HTLV and Related Viruses Montreal, Canada. 26-30 June 2013

HTLV-I associated adult T-cell leukemia/lymphoma (ATL) carries a dismal prognosis due to chemo-resistance and immuno-compromised micro-environment. The combination of zidovudine and interferon-alpha (IFN) significantly improved survival in ATL. Promising results were reported by adding arsenic trioxide to zidovudine and IFN. Here we assessed Th1/Th2/T $\mathrm{T}_{\text {reg }}$ cytokine gene expression profiles in 16 ATL patients before and 30 days after treatment with arsenic/IFN/zidovudine, in comparison with HTLV-I healthy carriers and sero-negative blood donors. ATL patients at diagnosis displayed a $\mathrm{T}_{\text {reg }} / \mathrm{Th} 2$ cytokine profile with significantly elevated transcript levels of Foxp3, interleukin-10 (IL-10), and IL-4 and had a reduced Th1 profile evidenced by decreased transcript levels of interferon- $\gamma($ IFN- $\gamma$ ) and IL-2. Most patients (15/16) responded, with $\mathrm{CD} 4{ }^{+} \mathrm{CD} 25^{+}$cells significantly decreasing after therapy, paralleled by decreases in Foxp3 transcript. Importantly, arsenic/IFN/zidovudine therapy sharply diminished IL-10 transcript and serum levels concomittant with decrease in IL-4 and increases in IFN- $\gamma$ and IL-2 mRNA, whether or not values were adjusted to the percentage of $\mathrm{CD} 4{ }^{+} \mathrm{CD} 25^{+}$cells. The observed shift from a $\mathrm{T}_{\text {reg }} / \mathrm{Th} 2$ phenotype before treatment toward a Th1 phenotype after treatment with arsenic/IFN/zidovudine may play an important role in restoring an immuno-competent micro-environment, which enhances the eradication of ATL cells and the prevention of opportunistic infections.

\section{Authors' details}

'Department of Biology, Faculty of Sciences, Lebanese University, Hadath, Lebanon. ${ }^{2}$ Microbiology and Virology Research Center, Bu-Ali Research institute, Mashhad University of Medical Sciences, Mashhad, Iran. ${ }^{3}$ Immunology Research Centre Bu-Ali Research Institute, Mashhad University of Medical Sciences, Mashhad, Iran. ${ }^{4}$ Lebanese American University, School of Arts and Sciences, Lebanon. ${ }^{5}$ Islamic University, Faculty of Nursing Sciences, Lebanon. ${ }^{6}$ Department of Internal Medicine, Mashhad University of Medical Sciences, Mashhad, Iran. 'Department of Internal Medicine, American University of Beirut, Beirut, Lebanon. ${ }^{8}$ Tehran University of Medical Sciences, Tehran, Iran. ${ }^{9}$ INSERM UMR 944 and CNRS UMR 7212, Hôpital Saint Louis, Paris, France. ${ }^{10}$ CNRS UMR 8147, Hôpital Necker, Paris, France.

Published: 7 January 2014

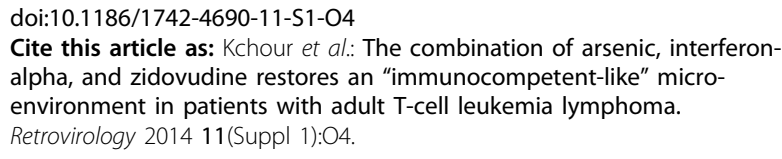

Cite this article as: Kchour et al:: The combination of arsenic, interferonalpha, and zidovudine restores an "immunocompetent-like" microenvironment in patients with adult T-cell leukemia lymphoma. Retrovirology 2014 11(Suppl 1):04. 